\title{
Phase III of Study of R-CHOP-21 vs R-CHOP-14 for Untreated Stage III and IV B-cell Non-Hodgkin's Lymphoma: a Report from Iran
}

\author{
Mehrdad Payandeh ${ }^{1}$,Safa Najafi ${ }^{2}$, Fateme-Zahra Shojaiyan ${ }^{2}$, Masoud Sadeghi $^{1 *}$
}

\begin{abstract}
Background: A combination of rituximab to cyclophosphamide, doxorubicin, vincristine, and prednisone (R-CHOP) is one of the most effective front-line therapies to treat B-cell non-Hodgkin's lymphoma (NHL). The aim of this trial was to evaluate overall survival (OS), progression free survival (PFS) and toxicity of R-CHOP-14 compared to R-CHOP-21 in untreated stage III and IV B-cell NHL patients with Iranian ethnicity. Materials and Methods: In phase III trial, patients with previously untreated stage III and IV indolent and aggressive B-cell NHL were randomly assigned by using a minimization method to receive six to eight cycles of either R-CHOP-21 (administered every 21 days) or R-CHOP-14 (administered every 14 days with granulocyte colony-stimulating factor). Results: A total of 143 patients were randomly enrolled in our study (66 patients in R-CHOP-14 group and 77 patients in R-CHOP-21), between 2011 and 2014. The mean follow-up was 45 months at the time of treatment analysis. The 2-year and 5-year PFS rates for the R-CHOP-14 group were 83.6\% vs $73.6 \%$ and for R-CHOP-21 group were $75 \%$ vs 54\%. The 2-year and 5-year OS rates for R-CHOP-14 group were $98 \%$ vs 89\% and for R-CHOP-21 group were $84.4 \%$ vs $67.5 \%$. There was a significant correlation for PFS and OS in the two arms. There was no significant difference between adverse events with the two regimens. Conclusions: In our research improved survival was found with CHOP-14 as compared to CHOP-21. It is possible that drug metabolism in different races/ethnicities may be one important factor.
\end{abstract}

Keywords: Non-hodgkin's lymphoma - phase III trial - R-CHOP - overall survival - progression-free survival

Asian Pac J Cancer Prev, 17 (3), 1513-1517

\section{Introduction}

Cyclophosphamide, doxorubicin, vincristine, and prednisone (CHOP) regimen is a standard treatment for patients with diffuse large B-cell (DLBCL) non-Hodgkin's lymphoma (NHL), and its 5-year overall survival (OS) rate is $30 \%-40 \%$. Rituximab is a chimeric monoclonal antibody directly against CD20-positive B cells, and has good effect on DLBCL-NHL (Lin et al., 2015). Combination of rituximab to CHOP (R-CHOP) is one of the most effective front-line therapies to treat B-cell NHL (Watanabe et al., 2011). The WHO classification of NHL included DLBCL, mantle cell lymphoma, follicular lymphoma, extranodal marginal zone lymphoma, Burkitt lymphoma, and B-cell lymphoma whose further types were unclassified (Kikuchi et al., 2015).

It was suggested that decreasing the treatment interval from three weeks (CHOP-21) to two weeks (CHOP-14) may improve survival and disease control of patients with aggressive lymphoma. R-CHOP-21 has remained the standard chemotherapy for aggressive non-Hodgkin's lymphoma (Vidal et al., 2015).

Granulocyte colony-stimulating factor (G-CSF) prevents myelotoxicity. Full dose CHOP with G-CSF, based on the leukocyte count oriented schedule, can be achieved with shortened intervals (Sawada et al., 1995; Itoh et al., 2002), an approach which will increase the quality of life for the patients by reducing the days of treatment as well as the cost of G-CSF (Sawada et al., 1995). Most patients with localized high-grade nonHodgkin's lymphoma (NHL) can be cured with or without adjuvant radiotherapy. High-dose CHOP followed by locoregional radiotherapy is a feasible treatment for localized high-grade NHL (Bernard et al., 2005).

The aim of this trial is to evaluate OS, PFS and toxicity of R-CHOP-14 compared to R-CHOP-21 in untreated stage III and IV B-cell NHL patients with Iranian ethnicity.

\section{Materials and Methods}

\section{Patients}

This trial was approved by Kermanshah University 
Ethics Committee, Kermanshah, Iran. In phase III study, patients with previously untreated stage III and IV indolent and aggressive B-cell NHL according to the WHO classification (Jaffe et al., 2001) were randomly assigned by using a minimization method to receive six to eight cycles of either R-CHOP-21 (arm A) or R-CHOP-14 ( $\operatorname{arm} \mathrm{B})$. A requirement for therapeutic intervention was not well defined and, consequently, some of the patients enrolled were treated immediately after diagnosis without watchful waiting. All patients gave written, informed consent before enrollment. R-CHOP-21 was intravenous cyclophosphamide $750 \mathrm{mg} / \mathrm{m}^{2}$, doxorubicin $50 \mathrm{mg} /$ $\mathrm{m} 2$, vincristine $1.4 \mathrm{mg} / \mathrm{m}^{2}$ (maximum dose $2 \mathrm{mg}$ ), and rituximab (MabThera $\left.{ }^{\circledR}\right) 375 \mathrm{mg} / \mathrm{m}^{2}$ on day 1 , and oral

Table 1. The baseline characteristics for the patients with Non-Hodgkin's Lymphoma based on R-CHOP regimens $(n=143)$

\begin{tabular}{|c|c|c|c|}
\hline Variables & $\begin{array}{c}\text { R- } \\
\text { CHOP-14 }\end{array}$ & R-CHOP-21 & P-value \\
\hline & $\mathrm{N}=66$ & $\mathrm{~N}=77$ & \\
\hline \multicolumn{4}{|l|}{ Age, year } \\
\hline Mean & 46.3 & 46 & $0.871 * *$ \\
\hline Range & $20-82$ & $16-78$ & \\
\hline$\leq 60$ & $17(25.7 \%)$ & $16(20.7 \%)$ & $0.306 \#$ \\
\hline$>60$ & $49(74.3 \%)$ & $61(79.3 \%)$ & \\
\hline \multicolumn{3}{|l|}{ Sex } & $0.421 \#$ \\
\hline Male & $40(60.6 \%)$ & $49(63.6 \%)$ & \\
\hline Female & $26(39.4 \%)$ & $28(36.4 \%) 0$ & \\
\hline \multicolumn{3}{|l|}{ Type of NHL } & $0.139 \#$ \\
\hline Nodal & $42(63.6 \%)$ & $41(53.2 \%)$ & \\
\hline Extra nodal & $24(36.4 \%)$ & $36(46.8 \%)$ & \\
\hline \multicolumn{3}{|l|}{ Subtype } & $0.158 \#$ \\
\hline Aggressive & $61(92.4 \%)$ & $68(88.3 \%)$ & \\
\hline Indolent & $5(7.6 \%)$ & $11(14.3 \%)$ & \\
\hline \multicolumn{4}{|c|}{ Ki-67 expression* } \\
\hline Mean & 59 & 55 & $0.112 * *$ \\
\hline$<65 \%$ & $28(42.4 \%)$ & $23(29.9 \%)$ & $0.269 \#$ \\
\hline$\geq 65 \%$ & $38(57.6 \%)$ & $54(70.1 \%)$ & \\
\hline \multicolumn{3}{|l|}{ Organomegaly } & $0.267 \#$ \\
\hline Yes & $4(6.1 \%)$ & $8(10.4 \%)$ & \\
\hline No & $62(93.9 \%)$ & $69(89.6 \%)$ & \\
\hline \multicolumn{3}{|c|}{ Lymphadenopathy } & $0.557 \#$ \\
\hline Yes & $37(56.1 \%)$ & $43(55.8 \%)$ & \\
\hline No & $29(43.9 \%)$ & $34(44.2 \%)$ & \\
\hline \multicolumn{3}{|c|}{ Adjuvant radiotherapy } & $0.527 \#$ \\
\hline Yes & $61(92.4 \%)$ & $72(93.5 \%)$ & \\
\hline No & $5(7.6 \%)$ & $5(6.5 \%)$ & \\
\hline
\end{tabular}

* Ki67 index was tested for 51 patients in R-CHOP-14 group and 36 patients in R-CHOP-21 group, **T-test, \#Chi-square test. R-CHOP$21=$ cycles of rituximab plus cyclophosphamide, doxorubicin, vincristine, and prednisolone every 21 days. R-CHOP-14=cycles of rituximab plus cyclophosphamide, doxorubicin, vincristine, and prednisolone every 14 days with granulocyte colony-stimulating factor prednisolone $100 \mathrm{mg} / \mathrm{m}^{2}$ on days $1-5$, administered every 21 days. R-CHOP-14 was intravenous cyclophosphamide $750 \mathrm{mg} / \mathrm{m}^{2}$, doxorubicin $50 \mathrm{mg} / \mathrm{m}^{2}$, vincristine $2 \mathrm{mg}$, rituximab(MabThera ${ }^{\circledR}$ ) $375 \mathrm{mg} / \mathrm{m}^{2}$ on day 1 , and oral prednisolone $100 \mathrm{mg}$ on days 1-5, administered every 14 days. G-CSF has been used in a few patients as secondary prophylaxis (based on the patient's need) in R-CHOP-21 group and has been used in all patients for prevention in R-CHOP-14 group. $92.4 \%$ patients received adjuvant radiotherapy in R-CHOP-14 group and $93.5 \%$ patients in R-CHOP-21 group. Age, sex, type of NHL, subtype, Ki-67 index, organomegaly, lymphadenopathy and radiotherapy, anatomic sites of NHL and recurrence were checked in all patients. Patients with cardiovascular, renal, hepatic disease (hepatitis B or hepatitis C) and T-cell lymphoma and also patients with initial neutrophil count $<1.5 \times 10^{9}$ per $\mathrm{L}$ and initial platelet count $<100 \times 10^{9}$ per L were excluded. 61 patients $(92.4 \%)$ were treated with radiotherapy after chemotherapy in $\mathrm{R}-\mathrm{CHOP}-14$ group and 72 patients $(93.5 \%)$ were treated with radiotherapy after chemotherapy in R-CHOP-21 group.

Patients were assessed before treatment; at each attendance for treatment; and then after treatment every 3 months until 1 year, then every 6 months until 2 years, and thereafter every year. Details of treatment and adverse effects, performance status, and results of blood counts and other reports were checked by clinicians. An immunohistochemical panel was done for all available

Table 2. Anatomic Sites for NHL and Recurrence Based Treatment Regimens

\begin{tabular}{lcc}
\hline Variables & R-CHOP-14 & R-CHOP-21 \\
\hline Anatomic site of NHL, $\mathrm{n}$ & 8 & 11 \\
$\quad$ GIT & 4 & 11 \\
Bone & 4 & 9 \\
Axillary LN & 14 & 11 \\
Cervical LN & 3 & 5 \\
Inguinal LN & 11 & 5 \\
Mediastenal LN & 2 & 4 \\
Abdominal LN & 3 & 3 \\
Muscle & 17 & 18 \\
Others & 66 & 77 \\
Total & & \\
Anatomic site of recurrence, n & 0 & 2 \\
GIT & 1 & 4 \\
Bone & 2 & 3 \\
Abdominal LN & 1 & 2 \\
Axillary LN & 3 & 5 \\
Inguinal LN & 3 & 2 \\
Cervical LN & 1 & 1 \\
Mediastenal LN & 0 & 2 \\
Supraclavicular LN & 2 & 2 \\
Gland & 5 & 1 \\
Brain & 1 & 2 \\
Lung & 19 & 25 \\
\hline Total & & \\
\hline
\end{tabular}

NHL, non-Hodgkin's lymphoma; GIT, gastrointestinal stromal tumors; LN, lymph node. R-CHOP-21=cycles of rituximab plus cyclophosphamide, doxorubicin, vincristine, and prednisolone every 21 days. R-CHOP-14=cycles of rituximab plus cyclophosphamide, doxorubicin, vincristine, and prednisolone every 14 days with granulocyte colony-stimulating factor 
Phase III Study of R-CHOP-21 vs R-CHOP-14 for Untreated Stage III and IV B-cell Non-Hodgkin's Lymphoma in Iran specimens and included CD20, CD99, CD15, CD3, CD2, Ki-67 expression.

\section{Statistical analysis}

Outcomes for this study were OS, PFS and toxicity. Comparison between OS and PFS for two arms was checked by GraphPad Prism 5 software that the log-rank test was used to compare the Kaplan-Meier curves for OS and PFS. Chi-square test and T-test in SPSS software were used for to compare the differences in the benefits of R-CHOP-14 in different patients sub-grouped according to baseline variables. Also, Cox proportional hazard regression analysis was used to check the effects of various parameters on the primary analysis. P-value $<0.05$ was considered as statistically significant.

\section{Results}

Between 2011 and 2014 from two centers, 143 patients were randomly enrolled ( 66 patients in R-CHOP-14 group and 77 patients in R-CHOP-21). The baseline variables were balanced in the patients for two groups (Table 1). The mean dose received for each drug by treatment group was similar in the R-CHOP-21 and R-CHOP-14 groups. Table 2 shows sites of NHL and recurrence for the patients in two arms.

The mean follow-up was 45 months at the time of treatment analysis. The 2-year PFS rate for R-CHOP-14

group was $83.6 \%$ vs. $75 \%$ for R-CHOP-21 group and also the mean PFS was 24 months for R-CHOP-14 group $v s$. 19 months for R-CHOP-21 group that different was no significant(hazard ratio [HR] 0.53, 95\% CI 0.22-1.30; $\mathrm{p}<0.1710$ ) (Figure 1).

The 2-year OS rate (the mean OS) for R-CHOP-14 group and R-CHOP-21 was 98\% (24 months) and $84.4 \%$ (19.5 months), respectively that different was significant (HR 0.19, 95\% CI 0.04-0.60; p<0.0062). The 5-year PFS rate for R-CHOP-14 group was 73.8\% vs. 54\% for R-CHOP-21 group and also the mean PFS was 24 months for R-CHOP-14 group vs. 19 months for R-CHOP-21 group that there was a significant difference between arms (HR 0.51, 95\% CI 0.29-0.89; p=0.0188). The 5-year OS rate (the mean OS) for R-CHOP-14 group and R-CHOP-21 was $98 \%$ (24 months) and $84.4 \%$ (19.5 months), respectively that there was no significant difference between arms (HR 0.33, 95\% CI 0.16-0.68; $\mathrm{p}<0.0027)$.

A Cox proportional hazard regression analysis was used to assess the effects of various parameters on the primary analysis. These factors did not affect the point estimate of the treatment arms. (Table 3) There were no unfavorable predictors for PFS or OS (Table 4).

Table 5 shows comparison of adverse events for two arms. Although neutropenia and fever were higher in R-CHOP-14 group, but there was no significant different between adverse events in two regimens $(\mathrm{P}>0.05)$.

Table 3. Multivariate Survival Analysis Using Cox's Regression Model for Affecting of Variables on Progression Free Survival

\begin{tabular}{lcc}
\hline Variables & P-value & HR* \\
\hline Treatment arm, R-CHOP-14 v R-CHOP-21 & 0.061 & 1.73 \\
Age (years), > 60 v $\leq 60$ & 0.873 & 1.05 \\
Sex, male v female & 0.692 & $0.975-3.069$ \\
Type, nodal v extra nodal & 0.172 & $0.576-1.916$ \\
Subtype, aggressive v indolent & 0.736 & $0.562-1.466$ \\
Ki-67 expression, <65\% v $\geq 65 \%$ & 0.657 & 0.87 \\
Organomegaly, yes v no & 0.383 & 1.127 \\
Lymphadenopathy, yes v no & 0.985 & 1.561 \\
Adjuvant radiotherapy, yes v no & 0.508 & 0.995 \\
\hline
\end{tabular}

*HRs are presented as the risk of the right-side category (ie, right side of $\mathrm{v}$ in Parameter column) to the left-side category (ie, left side of $\mathrm{v}$ ). Abbreviations: MFS, metastasis-free survival; HR, hazard ratio; NHL, non-Hodgkin's lymphoma. R-CHOP-21=cycles of rituximab plus cyclophosphamide, doxorubicin, vincristine, and prednisolone every 21 days. R-CHOP-14=cycles of rituximab plus cyclophosphamide, doxorubicin, vincristine, and prednisolone every 14 days with granulocyte colony-stimulating factor.

Table 4. Multivariate Survival Analysis Using Cox's Regression Model for Affecting of Variables on Overall Survival

\begin{tabular}{lcc}
\hline Variables & P-value & HR* \\
\hline Treatment arm, R-CHOP-14 v R-CHOP-21 & 0.349 & 1.326 \\
Age (years), > 60 v $\leq 60$ & 0.875 & 0.955 \\
Sex, male v female & 0.263 & 0.756 \\
Type, nodal v extra nodal & 0.342 & $0.537-1.699$ \\
Subtype, aggressive v indolent & 0.285 & $0.463-1.233$ \\
Ki-67 expression, <65\% v $\geq 65 \%$ & 0.704 & 0.634 \\
Organomegaly, yes v no & 0.321 & 0.907 \\
Lymphadenopathy, yes v no & 0.609 & 1.66 \\
Adjuvant radiotherapy, yes v no & 0.511 & $0.275-1.462$ \\
\hline
\end{tabular}

*HRs are presented as the risk of the right-side category (ie, right side of $\mathrm{v}$ in Parameter column) to the left-side category (ie, left side of v). Abbreviations: MFS, metastasis-free survival; HR, hazard ratio; NHL, non-Hodgkin's lymphoma. R-CHOP-21=cycles of rituximab plus cyclophosphamide, doxorubicin, vincristine, and prednisolone every 21 days. R-CHOP-14=cycles of rituximab plus cyclophosphamide, doxorubicin, vincristine, and prednisolone every 14 days with granulocyte colony-stimulating factor. 
Table 5. Grade 3 or 4 Adverse Events for Treatment Regimens

\begin{tabular}{lccc}
\hline Variables & R-CHOP-14 & R-CHOP-21 & P-value \\
\hline Neutropenia (\% grade 3,4) & $16(25.7 \%)$ & $12(15.8 \%)$ & $\mathrm{P}>0.05$ \\
Anemia (\% grade 3) & $6(9.1 \%)$ & $7(9.1 \%)$ & $\mathrm{P}>0.05$ \\
AST(\% grade 3) & $3(4.5 \%)$ & $3(3.9 \%)$ & $\mathrm{P}>0.05$ \\
ALT(\% grade 3) & $3(4.5 \%)$ & $4(5.2 \%)$ & $\mathrm{P}>0.05$ \\
Infection due to sepsis(\% grade 4) & $7(10.6 \%)$ & $7(9.1 \%)$ & $\mathrm{P}>0.05$ \\
Fever (\% grade 3, 4) & $17(25.8 \%)$ & $12(15.6 \%)$ & $\mathrm{P}>0.05$ \\
Thrombocytopenia $(\%$ grade 4) & $8(12.1 \%)$ & $9(11.7 \%)$ & $\mathrm{P}>0.05$ \\
\hline
\end{tabular}

Data are number (\%), R-CHOP-21=cycles of rituximab plus cyclophosphamide, doxorubicin, vincristine, and prednisolone every 21 days. R-CHOP$14=$ cycles of rituximab plus cyclophosphamide, doxorubicin, vincristine, and prednisolone every 14 days with granulocyte colony-stimulating factor.
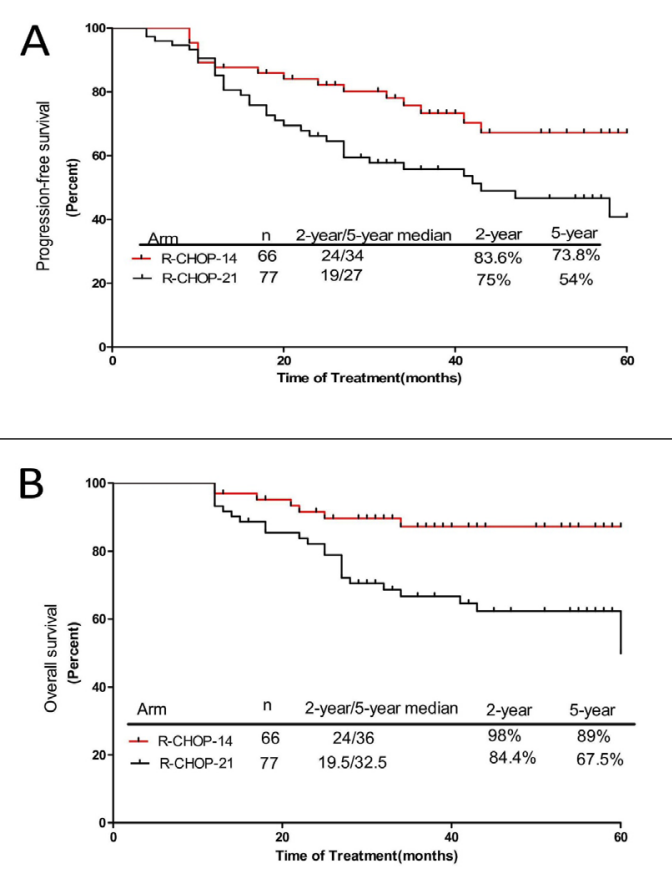

Figure 1.A) Progression-free Survival and (B) Overall Survival for Patients with B-cell non-Hodgkin's lymphoma. R-CHOP-14, rituximab plus cyclophosphamide, doxorubicin, vincristine, and prednisone (R-CHOP) administered every 2 weeks with granulocyte colony-stimulating factor; R-CHOP-21, R-CHOP administered every 3 weeks

\section{Discussion}

Immunochemotherapy with R-CHOP has become the standard of care for elderly patients with DLBCL (Delarue et al., 2013). In a study, 1080 patients were assigned to R-CHOP-21 or R-CHOP-14 with G-CSF that around half of cases received radiotherapy after chemotherapy in every arm, 2-year OS was more in R-CHOP-14 group than R-CHOP-21 group and also 2-year PFS was better in R-CHOP-14 than R-CHOP-21, but there was no significant correlation between OS and PFS in two arms. Grade 3 or 4 neutropenia was higher in the R-CHOP-21 group ( $60 \%$ v $31 \%$ ), grade 3 or 4 thrombocytopenia was higher with R-CHOP-14 (9\% v 5\%) and infection (23\% $\mathrm{v} 18 \%$ ). Frequencies of non-hematological adverse events were similar in the R-CHOP-21 and R-CHOP-14 groups (Cunningham et al., 2013). Three-hundred patients in Watanabe's research (1) with untreated stages III to IV indolent B-cell lymphoma were randomly assigned to six cycles of R-CHOP-21 or R-CHOP-14 with G-CSF. There was no significant difference in PFS between arms (the median was 3.7 (R-CHOP-21) v 4.7 years (R-CHOP-14)). Also, PFS rate was $57 \%$ v $58 \%$ at 3 years and $41 \%$ v $43 \%$ at 6 years, respectively. Also, 6-year OS was $87 \%$ [R-CHOP-21] v 88\% [R-CHOP-14]. There was no significant correlation between two groups for OS or PFS. Although grade 4 neutropenia and grade 3 infections were more frequent in the R-CHOP-21 group, R-CHOP was feasible in both arms (Watanabe et al., 2011). A Japanese research (Ohmachi et al., 2011), reported that eight-year OS and PFS rates were more in R-CHOP-21 compared to R-CHOP-14, but no substantial differences were observed in between two arms. The 3-year PFS was 58\% for patients treated with R-CHOP-21 in a study matched that for the control patients in the Primary Rituximab and Maintenance (PRIMA) study (Salles et al., 2011). Hiddemann (2005) reported that grade 4 neutropenia and grade 3 infection occurred more often during R-CHOP-21 than during R-CHOP-14. Although a total 50 patients in R-CHOP-21 group (40\%) received G-CSF. In 1829 adults receiving $( \pm \mathrm{R}) \mathrm{CHOP}-14$ or $( \pm \mathrm{R}) \mathrm{CHOP}-21$. Overall, $33 \%$ of patients were anemic during chemotherapy (Haioun et al., 2011). In patients aged 60-80 years, R-CHOP given every 14 days did not demonstrate survival advantage over R-CHOP 21 (Armitage,2015; Delarue et al., 2009), that 3-year event-free survival was 56\% in the R-CHOP14 group and $60 \%$ in the R-CHOP2 1 group and the frequency of toxic side-effects was similar between regimens(Grade 3-4 neutropenia occurred in $74 \%$ patients allocated R-CHOP14 and 64\% assigned R-CHOP21) (Delarue et al., 2013). In others hand, a research reported that recent phase III trials have demonstrated improved survival by modifying CHOP either through adding rituximab or shortening the time between cycles to 14 days (Halaas et al., 2005). Therefore, adding the immunotherapeutic agent rituximab to either $\mathrm{CHOP}$ has been shown to improve outcomes significantly, such that six cycles of $\mathrm{R}-\mathrm{CHOP}$ plus two cycles of rituximab are as effective as eight cycles of R-CHOP, and R-CHOP-21 appears to be at least as effective as the more dose-intense R-CHOP-14 (Gisselbrecht, 2011). Six courses of R-CHOP 21 with radiotherapy to the sites of previous bulky disease was shown to be effective in this group of patients (Pfreundschuh et al., 2006). In this trial, OS and PFS were better in R-CHOP-14 group with G-CSF and two cycles rituximab compared to R-CHOP-21 and two cycles rituximab. Almost more patients were treatment with 
Phase III Study of R-CHOP-21 vs R-CHOP-14 for Untreated Stage III and IV B-cell Non-Hodgkin's Lymphoma in Iran

adjuvant chemotherapy in two arms. There was significant correlation between them and also percentage of adverse events were almost similar in two arms and very lower than other studies that also Cunningham (2013) showed that R-CHOP-14 had efficacy in patients with DLBCL and Halaas (2005) reported that R-CHOP-14 was feasible and early results showed favorable efficacy compared to $\mathrm{R}-\mathrm{CHOP}-21$, similar to German group for $\mathrm{CHOP}$ regimen (Pfreundschuh et al., 2004). Our study was done in patients with Iranian ethnicity that Wang (2008) documented the impact of racial and ethnic disparities in access to treatment for DLBCL that could increase disparities in outcomes such as survival in the presence of R-CHOP. Also, Levi (2013) reported that affecting of treatment in NHL patients may be different in ethnic groups.

In conclusion, In our research that why the improved survival reported with CHOP-14 like German group is still uncertain, but it is possible that drug metabolism in different races/ethnicities be one important factor. Also, adverse events in two our arms were very lower than other studies.

\section{Acknowledgements}

This research was supported by Roche Company, This study was registered at http://www.irct.ir(registration number IRCT138903181617N3.).

\section{References}

Armitage JO (2012). My treatment approach to patients with diffuse large B-cell lymphoma. Mayo Clin Proc, 87, 161-71.

Bernard M, Cartron G, Rachieru P, et al (2005). Long-term outcome of localized high-grade non-Hodgkin's lymphoma treated with high dose CHOP regimen and involved field radiotherapy: results of a GOELAMS study. Haematologica, 90, 802-9.

Cunningham D, Hawkes EA, Jack A, et al (2013). Rituximab plus cyclophosphamide, doxorubicin, vincristine, and prednisolone in patients with newly diagnosed diffuse large B-cell non-Hodgkin lymphoma: a phase 3 comparison of dose intensification with 14-day versus 21-day cycles. Lancet, 381, 1817-26.

Delarue R, Tilly H, Mounier N, et al (2013). Dose-dense rituximab-CHOP compared with standard rituximab-CHOP in elderly patients with diffuse large B-cell lymphoma (the LNH03-6B study): a randomised phase 3 trial. Lancet Onco, 14, 525-33.

Delarue R, Tilly H, Mounier N, et al (2009). R-CHOP14 compared to R-CHOP21 in elderly patients with diffuse large B-cell lymphoma: results of the interim analysis of the LNH03-6B GELA study. Blood, 114, 52.

Gisselbrecht C (2011). Current approaches to the treatment of non-Hodgkin's lymphoma. Hematol Rep, 28, 3.

Haioun C, Salar A, Pettengell R, et al (2011). Anemia and erythropoiesis-stimulating agent administration in patients with non-Hodgkin lymphoma treated with cyclophosphamide, doxorubicin, vincristine, and prednisolone \pm rituximab chemotherapy: results from an observational study. Leuk Lymphoma, 52, 796-803.

Halaas JL, Moskowitz CH, Horwitz S, et al (2005). R-CHOP-14 in patients with diffuse large B-cell lymphoma: feasibility and preliminary efficacy. Leuk Lymphoma, 46, 541-7.

Hiddemann W, Kneba M, Dreyling M, et al (2005). Frontline therapy with rituximab added to the combination of cyclophosphamide, doxorubicin, vincristine, and prednisone (CHOP) significantly improves the outcome for patients with advanced-stage follicular lymphoma compared with therapy with CHOP alone: Results of a prospective randomized study of the German Low-Grade Lymphoma Study Group. Blood, 106, 3725-32.

Itoh K, Ohtsu T, Fukuda H, et al (2002). Randomized phase II study of biweekly CHOP and dose-escalated CHOP with prophylactic use of lenograstim (glycosylated G-CSF) in aggressive non-Hodgkin's lymphoma: Japan Clinical Oncology Group Study 9505. Ann Oncol, 13, 1347-55.

Jaffe ES, Harris NL, Stein H, et al (2001). World Health Organisation Classifi cation of Tumours. Pathology and genetics of tumours of haematopoietic and lymphoid tissues. Lyon: IARC Press.

Kikuchi M, Nakasone H,Akahoshi Y, et al (2015). Reduced-dose (two-thirds) R-CHOP chemotherapy for elderly patients with non-Hodgkin lymphoma. J Chemother, 27, 99-105.

Levi I, Feuchtwanger M, Rabinovich A, et al (2013). Clinical and epidemiologic characteristics of non Hodgkin's lymphoma in Bedouins in the south of Israel. Springerplus, 2, 672.

Lin TY, Zhang HY, Huang Y, et al (2005). [Comparison between $\mathrm{R}-\mathrm{CHOP}$ regimen and $\mathrm{CHOP}$ regimen in treating naive diffuse large B-cell lymphoma in China--a multi-center randomized trial]. Ai Zheng, 24, 1421-6.

Ohmachi K, Tobinai K, Kobayashi Y, et al (2011). Phase III trial of CHOP-21 versus CHOP-14 for aggressive non-Hodgkin's lymphoma: final results of the Japan clinical oncology group study, JCOG 9809. Ann Oncol, 22, 1382-91.

Pfreundschuh M, Trümper L, Kloess M, et al (2004). Twoweekly or 3-weekly CHOP chemotherapy with or without etoposide for the treatment of elderly patients with aggressive lymphomas: results of the NHL-B2 trial of the DSHNHL. Blood, 104, 634-41.

Pfreundschuh M, Trümper L, Osterborg A, et al (2006). CHOPlike chemotherapy plus rituximab versus CHOP-like chemotherapy alone in young patients with good-prognosis diffuse large-B-cell lymphoma: a randomised controlled trial by the MabThera International Trial (MInT) Group. Lancet Oncol, 7, 379-91.

Salles G, Seymour JF, Offner F, et al (2011). Rituximab maintenance for 2 years in patients with high tumour burden follicular lymphoma responding to rituximab plus chemotherapy (PRIMA): A phase 3, randomised controlled trial. Lancet, 377, 42-51.

Sawada KI, Sato N, Kohno M, et al (1995). Efficacy of delayed granulocyte colony-stimulating factor after full dose CHOP therapy in non-Hodgkin's lymphoma: a pilot study for a leukocyte count oriented regimen. Leuk Lymphoma, 20, 103-9.

Vidal L, Shpilberg O, Gurion R, et al (2015). CHOP-like-14 compared to CHOP-like-21 for patients with aggressive lymphoma - a meta-analysis of randomized controlled trials. Acta Oncol, 1-8.

Wang M, Burau KD, Fang S, et al (2008). Ethnic variations in diagnosis, treatment, socioeconomic status, and survival in a large population-based cohort of elderly patients with non-Hodgkin lymphoma. Cancer, 113, 3231-41.

Watanabe T, Tobinai K, Shibata T, et al (2011). Phase II/III study of R-CHOP-21 versus R-CHOP-14 for untreated indolent B-cell non-Hodgkin's lymphoma: JCOG 0203 trial. J Clin Oncol, 29, 3990-8. 